\title{
An Expert System Based on Parametric Net to Support Motor Pump Multi-Failure Diagnostic
}

\author{
Flavia Cristina Bernardini and Ana Cristina Bicharra Garcia and Inhaúma \\ Neves Ferraz
}

\author{
ADDLabs - Active Documentation Design Laboratory, \\ UFF - Universidade Federal Fluminense \\ Av. Gal. Milton Tavares de Souza, s/nº - Campus da Praia Vermelha, Boa Viagem, Niterói, \\ RJ, Brazil. E-mail: flavia @addlabs.uff.br
ADDLabs - Active Documentation Design Laboratory
IC - Instituto de Computação
UFF - Universidade Federal Fluminense
Av. Gal. Milton Tavares de Souza, s/nº - Campus da Praia Vermelha, Boa Viagem, Niterói, RJ, Brazil. E-mail: bicharra@ic.uff.br

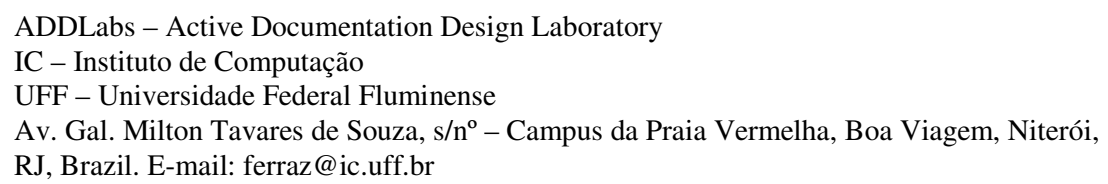

\begin{abstract}
Early failure detection in motor pumps is an important issue in prediction maintenance. An efficient condition-monitoring scheme is capable of providing warning and predicting the faults at early stages. Usually, this task is executed by humans. The logical progression of the condition-monitoring technologies is the automation of the diagnostic process. To automate the diagnostic process, intelligent diagnostic systems are used. Many researchers have explored artificial intelligence techniques to diagnose failures in general. However, all papers found in literature are related to a specific problem that can appear in many different machines. In real applications, when the expert analyzes a machine, not only one problem appears, but more than one problem may appear together. So, it is necessary to propose new methods to assist diagnosis looking for a set of occurring fails. For some failures, there are not sufficient instances that can ensure good classifiers induced by available machine learning algorithms. In this work, we propose a method to assist fault diagnoses in motor pumps, based on vibration signal analysis, using expert systems. To attend the problems related to motor pump analyses, we propose a parametric net model for multi-label problems. We also show a case study in this work, showing the applicability of our proposed method.
\end{abstract}

Please use the following format when citing this chapter:

Bernardini, F.C., Garcia, A.C.B. and Ferraz, I.N., 2009, in IFIP International Federation for Information Processing, Volume 296; Artificial Intelligence Applications and Innovations III; Eds. Iliadis, L., Vlahavas, I., Bramer, M.; (Boston: Springer), pp.13-20. 


\section{Introduction}

Motor pump fails detection is an important issue in prediction maintenance. Traditional maintenance procedures in industry have taken two routes. The first is to perform fixed time interval maintenance, and the second is to simply react to the plant failure as and when it happens. However, predictive maintenance through condition monitoring has become a new route to maintenance management. Researchers have studied a variety of machine faults, such as, unbalanced stator and rotor parameters, broken rotor bars, eccentricity and bearing faults, and different methods for fault identification have been developed [10]. These different methods are used effectively to detect the machine faults at different stages using different machine variables, such as current, temperature and vibrations. An efficient condition-monitoring scheme is capable of providing warning and predicting the faults at early stages. Monitoring systems obtain information about the machine in the form of primary data. Through the use of modern signal processing and analysis techniques, it is possible to give vital diagnostic information to equipment operator before it catastrophically fails. The problem with this approach is that the results require constant human interpretation. The logical progression of the condition-monitoring technologies is developing methods and tools to guide and, as a next stage of validation and improvement of the classification models, automate the diagnosis process. To this end, intelligent systems are used.

Recent artificial intelligent techniques, such as neural networks, fuzzy logic, expert system and genetic algorithm, have been employed to assist the diagnostic task to correctly interpret the fault data. Many researchers have explored these techniques to diagnose faults in induction motors, motor rolling bearings, and so on $[4,5,13,6]$. These papers describe diagnosis methods based on characteristics extraction and neural networks models induction for each separated fail. However, all papers found in literature are related to a specific problem that can appear in many different machines. In real applications, when the expert analyses a machine, not only one problem appears, but more than one problem may also appear. Another important characteristic of this problem is that different features of the collected signals are analyzed for each problem [7]. For this reason, it is necessary to propose new methods to assist diagnosis looking for many possible fails that can appear together.

There are many peculiar characteristics in machinery diagnostic problems that turns it an interesting research problem from machine learning and pattern recognition perspective. First, there are many signals captured from a machine that should be analyzed, which means that the method to be proposed to treat all the problems together should (a) be able to treat a large number of features, or (b) preprocess the data to predict the fails. Second, problems which an instance can be labeled with more than one class are called multi-label problems, and the development of methods in artificial intelligence to treat this kind of problem is still in research $[12,9,11,2]$. Third, to apply machine learning algorithms, such as artificial neural networks, support vector machines, and so on, it is necessary to have 
labeled instances of the domain to extract the model and predict future labels. In machinery failures, there are some fails that real instances can easily be obtained, e.g. unbalance and misalignment; but there are some that cannot and can seriously damage the machine, e.g. cavitation and bearing problems. Unbalance problems turns difficult to induce good classifiers [1]. All these reasons let us put a lot of effort to try to solve early diagnostic of motor pump failures problem.

In this work, we propose a method to assist fault diagnoses in motor pumps using expert systems, based on vibration signal analysis. Since the number of signals to be analyzed is large and, for some fails, there are not instances available to use machine learning algorithms, we decided to investigate a method to construct a parametric net model, based on expert knowledge. The main advantage of this method is that the model can explain its decisions by showing what was the reason followed by the parametric net to fails (labels) suggestion. To show the usability of our proposal, we describe a case study where we show the expert analyses and the results obtained by our proposed method in a specific type of motor pump.

This paper is organized as follows. Section 2 briefly describes how failures appear in vibration signals. Section 3 proposes a parametric net model to multi-label problems. Section 4 describes the parametric net model proposed for motor pump failure diagnosis. Finally, Section 5 concludes this work.

\section{Vibration Signal Analysis}

Motor pumps, due to the rotating nature of their internal pieces, produce vibrations. Accelerometers strategically placed in points next to the motor and the pump allows acceleration of the machine over time to be measured, thus generating a signal of the vibration level. Fig. 1 shows a typical positioning configuration of accelerometers on the equipment. In general, the orientations of the sensors follow the three main axes of the machine, e.g. vertical, horizontal and axial.

Fig. 1. Motor pump with extended coupling between motor and pump. The accelerometers are placed along the main directions to capture specific vibrations of the main axes. ( $\mathrm{H}=$ horizontal, $\mathrm{A}=$ axial, $\mathrm{V}=$ vertical)

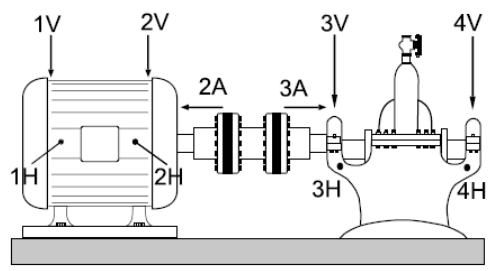

The presence of any type of machine faults causes change in mechanical and electrical forces that are acting in the machine [10]. The degree of change depends upon the nature and intensity of the fault. The change in machine vibration is the excitation of some of the vibration harmonics. Some of machine faults can be directly related to the vibration harmonic. Table 6.0, "Illustrated Vibration Diagnostic Chart", in [7], shows how to analyze signals, searching for mechanical and 
electrical faults. In what follows, electrical and mechanical faults are briefly described.

\section{A Parametric Net Model Proposed for Multi-label Problems}

Until the late ' 80 s the most popular approach to classification problems was a knowledge engineering one, consisting in manually defining a set of rules encoding expert knowledge on how to classify documents under the given categories. In the $90 \mathrm{~s}$, this approach has increasingly lost popularity in favor of the machine learning paradigm, according to with a general inductive process automatically builds a general hypothesis to classify new instances, based on instances previously labeled by some domain expert $[9,8]$. However, there are some problems that label attributed to the instances are not $100 \%$ guaranteed that are true, or there are unbalanced classes, which difficult the induction model process [1]. In these cases, it is interesting to construct an expert system, which contains the knowledge of the expert domain, represented in a parametric net, to (a) classify new instances with a set of labels; and (b) validate the available instances.

Parametric nets are used to inference logical facts, supporting decision making. In a parametric net, the parameters represent the problem features, domain properties, or decisions that must have made during the reasoning process. The various parameters of a knowledge base are inter-connected. These are directed connections, because they represent the dependency between parameters and define the logic precedence of the parameters instantiation. The parameter values represent the actual state of the problem being solved.

In its basic version, proposed in [3] to Active Document Design (ADD), and illustrated in Fig. 2, the parameters belong to one of three categories: primitive, derived or decision. Primitive parameters normally are the representation of the problem requisites. In general, these values are informed by the user during the reasoning process. Values of derived parameters are calculated based on values of other parameters. A value is chosen to a decided parameter from a set of alternatives of the attribute. The set of alternatives is filtered by constraints that represent conditions to be satisfied by values that come from the parameters connected to the decided parameter. The constraints are represented by rules. The rules has the form "if $<$ body $>$ then update weight $w_{k}$ with (positive or negative) value", where $<$ body $>$ is a set of conditions as primitive <operator $>$ value, and <operator $>$ may be $>,<, \leq, \geq$ and $=$. At the end of the reasoning process, all the alternatives are compared, and one alternative is chosen as an answer to the problem being solved. One common way to decide what is the best alternative is weighting each alternative. An evaluated criterion represents the value to be added to an alternative. The alternative with the maximum weight at the end of the evaluation criteria is selected as the best alternative. 
Fig. 2 . Sample of a parametric net. Dot lines represent link between primitives and derived parameters; regular lines represent link between one primitive or derided parameter and another decided parameter. Derived parameters are optional. Values are set into primitives.

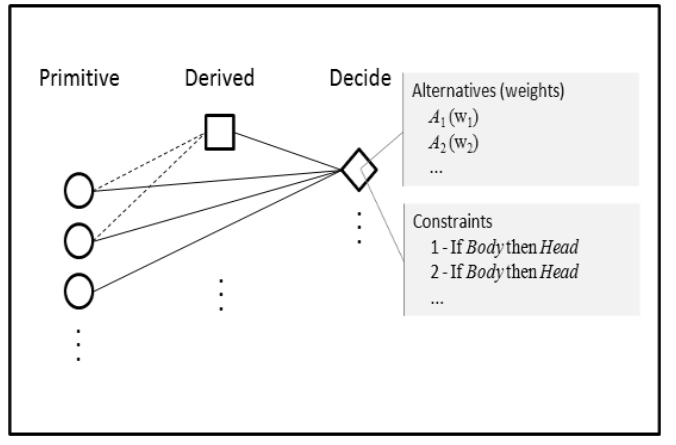

In domains where the features can be seen as different instantiations of a set of abstract features, each instance may increment or decrement the weight of an alternative. For example, in machine analyses using vibration signal, each signal captured in one different position of the machine is an instantiation of the possible vibration harmonics used to analyze the machine, and the intensity of each vibration harmonic value in each signal may reinforce or weaken an alternative. Fig. 3 illustrates how the signals are instances of abstract features. So, to adapt the model to offer more than one alternative, each abstract feature set instantiation is shown to the model, which may influence positively or negatively the alternatives. Again, in vibration analyses, each signal in frequency domain is pre-processed, and values related to each important vibration harmonic are extracted. Then, each set of vibration harmonic values, extracted from each signal, is shown to the model, and each alternative weight may be updated if the values obey the set of constraints. At the end of the process, all alternatives with positive weights are considered as possible classes, offering multi-label classification. The positive weights of each class can be normalized to the interval 0 to 1 , and values from 0 to 1 are related to each alternative, which can be used to help the expert to decide what the best class (alternative) is.

\section{A Parametric Net Model Constructed to Assist Motor Pumps Failure Diagnostic}

Model Construction: The type of motor pump considered in our study has the following characteristics: horizontal centrifuge with one stage (one rotor), direct coupling without gear box, and actuated by AC induction squirrel cage motor. The faults considered in our study are unbalance, misalignment, electric, hydraulic, cavitation, turbulence, bearing faults, looseness and resonance. Fig. 1 shows the points on motor pump where specific vibrations are captured. On each point, a time signal is captured by an accelerometer, and signal operators are applied to obtain acceleration, velocity and envelope signals. Each signal is important to detect groups of faults, or specific faults. These pieces of information about motor pump 


\begin{tabular}{|c|c|c|c|c|c|c|}
\hline & $\begin{array}{c}A F_{1} \\
(1 \mathrm{XRF})\end{array}$ & $\begin{array}{c}A F_{2} \\
(1 \mathrm{XRF})\end{array}$ & $\begin{array}{c}A F_{3} \\
(1 \mathrm{XRF})\end{array}$ & $\cdots$ & $\begin{array}{r}A F_{M} \\
(\ldots)\end{array}$ & \\
\hline$F V_{1}$ & $x_{1}$ & $x_{2}$ & $x_{3}$ & $\ldots$ & $x_{M}$ & $(1 \mathrm{H}-\mathrm{V} \times \mathrm{F})$ \\
\hline$F V_{2}$ & $x_{M+1}$ & $x_{M+2}$ & $x_{M+3}$ & $\ldots$ & $x_{2 M}$ & $(1 \mathrm{~V}-\mathrm{AxF})$ \\
\hline$F V_{3}$ & $x_{2 M+1}$ & $x_{2 M+2}$ & $x_{2 M+3}$ & $\ldots$ & $x_{3 M}$ & $(1 \mathrm{~A}-\mathrm{ExF})$ \\
\hline & $\ldots$ & $\ldots$ & $\ldots$ & $\ldots$ & $\ldots$ & $\ldots$ \\
\hline$F V_{T}$ & $x_{(T-1) M+1}$ & $x_{(T-1) M+2}$ & $x_{(T-1) M+3}$ & $\ldots$ & $x_{T M}$ & $\cdot$. \\
\hline
\end{tabular}

Fig. 3 . Instances of abstract features. Texts in parentheses comprehend an example of abstract features. In this case, the abstract features are vibration values in harmonic frequencies, e.g. $1 \mathrm{XRF}$ means vibration value in $1 \mathrm{x}$ rotation frequency, $2 \mathrm{XRF}$ means vibration value in $2 \mathrm{x}$ rotation frequency, and so on. The feature instances are signals $(\mathrm{VxF}$ - velocity per frequency signal; $\mathrm{AxF}$-acceleration per frequency signal; ExF - envelope per frequency value) captured on motor pump points $(1,2,3$ and 4$)$ at different directions $(\mathrm{H}$ - horizontal, $\mathrm{V}$ - vertical or $\mathrm{A}$ - axial).

diagnoses vibration are extracted from Table 6.0, "Illustrated Vibration Diagnostic Chart", in [7], and were explained and detailed by domain experts. All of this knowledge was used to construct constraints of the failure decision parameter. The parametric net model that aims to classify signal sets into a set of classes has one decision and many primitive parameters. Each fault is an alternative of the failure decision parameter of the parametric net.

Since vibration harmonics are what influence each alternative, each one is a primitive parameter. The primitives considered are vibration values in harmonic $(1 \mathrm{X}, 2 \mathrm{X}, \ldots)$ and inter-harmonic $(0.5 \mathrm{X}, 1.5 \mathrm{X}, \ldots)$ of the rotational frequency in r.p.s; RMS calculated in harmonic and inter-harmonic frequencies; BPFO, BPFI, BSF and RHF frequencies; electrical frequency; and pole frequency. Also, there are primitives that give to the model characteristics of the capturing position: velocity, acceleration, envelope, radial, axial, motor and pump, and they are set to true or false depending on the signal. E.g., if the signal is captured in position $1 \mathrm{~V}$ and is the velocity signal which is being analyzed, then velocity is set to true, where as acceleration and envelope are set to false; radial is set to true ${ }^{1}$ where as axial is set to false; and motor is set to true where as pump is set to false.

Model Application: To analyze a motor pump, ten acceleration signals in frequency domain are captured (one signal per point). Applying the mentioned operators, 30 signals are obtained. The model has all alternative weights initialize with 0 . Each velocity, acceleration and envelope signal of each point is shown to the model, which may increment the weight of each alternative. At the end of this process, all alternatives that have positive weights (greater than zero) are normalized to the range $0-1$, which are shown to the analyst.

A Case Study: We implemented a computational system, called ADDRPD, to help the analyst in all of the analysis process. Time signals of a specific motor pump are imported to the system. All transformations are applied and the resulting signals are shown to the user. One instance was labeled by the expert having only

${ }^{1}$ Signals read in $\mathrm{H}$ (horizontal) position are also considered radial. 
one problem: unbalance. However, when the parametric net was applied to the instance, three faults were diagnosed: unbalance (0.46), misalignment (0.44) and looseness (0.10). When showed to the expert domain, he explained that it is true that the three problems appears in the machine, however unbalance is the most problematic fault in that case. The expert analysis was basically based on Fig. 4.

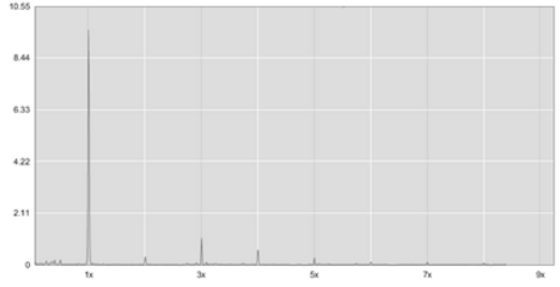

(a)

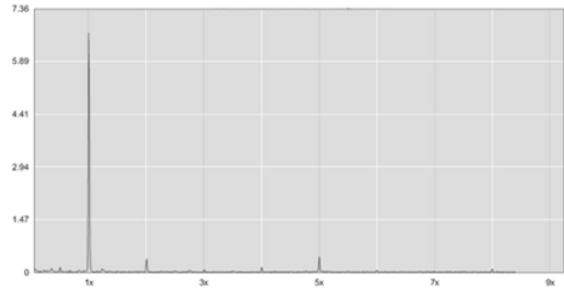

(b)

Fig. 4 . Velocity (RMS/s) per rotational frequency harmonics signals, showing high vibration and lower peaks in rotational frequency harmonics (a) captured at $1 \mathrm{H}$ and (b) captured at $2 \mathrm{~A}$.

Both signals showed in Fig. 4 are velocity per frequency signals, however Fig. 4 (a) was captured at radial direction, where as Fig. 4 (b) was captured at axial direction. So, since both has high peak vibration at $1 \mathrm{X}$, this represents that unbalance is the most representative failure in the motor pump. But the signal Fig. 4 (b) is from axial direction, which highly indicates misalignment failure, and the lower peaks at harmonic frequencies weakly indicate looseness. The analysis shows that the parametric net join to visual tools are efficient ways of analyzing motor pumps to diagnostic their failures.

\section{Conclusions and Future Work}

We propose in this work a method to assist fault diagnosis using parametric nets to represent the expert knowledge, based on vibration analysis. To this end, we propose a parametric net to multi-label problems. We present a model we developed for a special type of motor pump - horizontal centrifuge with one stage (one rotor), direct coupling without gear box, and actuated by $\mathrm{AC}$ induction squirrel cage motor. To preliminary evaluate the proposed model, we present a case study, using signals captured from a motor pump in use in real world. We could notice that the model could assist the expert interpretation of the signals.

Our method was implemented in a computational system called ADDRPD. The system will help to classify new instances, which we intend to improve our method. Ongoing work includes evaluating our method using recall and precision measure for each class, as a multi-label problem [12]. After validating (part of) the dataset, the parametric model will be updated and machine learning algorithms will be used to induce models and compare to the method proposed in this work. 


\section{References}

1. Batista, G.; Prati, R. C.; Monard, M. C. A study of the behavior of several methods for balancing machine learning training data. SIGKDD Explorations, 6(1):20-29, 2004.

2. Brinkler, K; Hullermeier, E. "Case-Based Multilabel Ranking". In: Proceedings 20th International Conference on Artificial Intelligence (IJCAI '07), pp. 702-707 (2007).

3. Garcia, A. C. B. "Active Design Documents: A New Approach for Supporting Documentation in Preliminary Routine Design", PhD thesis, Stanford University, (1992).

4. Kowalski, C. T.; Orlowska-Kowalska, T. "Neural networks application for induction motor faults diagnosis". Mathematics and Computers in Simulation, 63:435-448, 2003.

5. Li, B.; Chow, M.; Tipsuwan, Y; Hung, J.C. "Neural-Network-Based Motor Rolling Bearing Fault Diagnosis". IEEE Transactions on Industrial Electronics, 47(5), 2000.

6. Mendel, E.; Mariano, L. Z.; Drago, I.; Loureiro, S.; Rauber, T. W.; Varejão, F. M.; Batista, R.J. "Automatic Bearing Fault Pattern Recognition Using Vibration Signal Analysis". In: Proceedings IEEE International Symposium on Industrial Electronics (ISIE'08), Cambridge, pp. 955-960 (2008).

7. Mitchell, J. S. Introduction to Machinery Analysis and Monitoring, PenWel Books, Tulsa (1993).

8. Mitchell, T. Machine Learning. McGraw Hill (1997).

9. Sebastiani, F. "Machine learning in automated text categorization". ACM Computing Surveys. 34(1):1-47, 2002.

10. Singh, G.K.; Kazzaz, S. A. S. A. "Induction machine drive condition monitoring and diagnostic research - a survey". Electric Power Systems Research, 64:145-158, 2003.

11. Schapire, R. E.; Singer, Y.. "BoosTexter: A boosting-based system for text categorization". Machine Learning, 39(2/3):135-168, 2000.

12. X. Shen, M. Boutell, J. Luo, and C. Brown "Multi label Machine learning and its application to semantic scene classification". In: Proceedings of the 2004 International Symposium on Electronic Imaging (EI 2004), pp. 18-22 (2004).

13. Zhang, S.; Ganesan, R.; Xistris, G. D. "Self-Organizing Neural Networks for Automated Machinery Monitoring Systems". Mechanical Systems and Signal Processing, 10(5):517$532,1996$. 